\title{
Adsorption of Flexible Polymer by Poles
}

\author{
Jyoji MuraKami \\ Department of Applied Physics, Faculty of Engineering, \\ University of Tokyo, Bunkyo-ku, Tokyo 113, Japan.
}

(Received April 1, 1975)

\begin{abstract}
The adsorption of flexible polymer by distributed poles is investigated by the matrix method. The regularly arranged case and the randomly arranged case are formulated. In the latter case the secular equation is easily solved and various average quantities can be calculated as a function of the pole concentration.
\end{abstract}

KEY WORDS Adsorption / Flexible Polymer / Matrix Method / Pole Concentration /

Macromolecules in biological systems and in colloidal solutions interact with the surrounding materials, and so the physical effects of these interactions on the behavior and properties of the macromolecules are of importance and interest. Among the many kinds of interactions the physical adsorption is the simplest in nature; we encounter it most frequently, and it provides us with interesting statistical mechanical problems. In consequence, this macromolecular adsorption has been subjected to a number of investigations over the last 20 years. ${ }^{1-10}$

In these reports much attention has been paid to the configurational properties of the polymers near a planar surface, that is, to how the two competing effects, i.e., the decrease of the energy of the polymers due to the adsorption and the increase of the entropy by desorption, determine the characteristic dimensions of the adsorbed polymers.

However, in real systems the material that adsorbs the polymers is not necessarily the plane wall. It may be of a different geometrical shape or be subjected to some geometrical and/or physicochemical limitations.

In this respect two previous investgations on the adsorption of flexible polymers are noteworthy. One is the adsorption by a long rigid rod-like macromolecule investigated by Rubin ${ }^{12}$ and the other is that by the two parallel plane surfaces investigated by DiMarzio and Rubin. ${ }^{11}$ These model studies are interesting because membrane systems or colloidal solutions corresponding to these models are extensively found in nature.
In the present paper we treat the adsorption of the flexible polymer by the distributed rigid rod-like macromolecules (these are called "poles" hereafter) as an extension of the theory of Rubin. Here, as distinct from the theory of Rubin, we consider many poles and our interest lies in the dependence of the configurational properties on the concentration (or interpole distance) of the poles.

In $\S 1$ and $\S 2$ a lattice model of polymers (a simple cubic lattice is assumed for simplicity) is considered. The lattice constant is taken to be the same as the link length between adjacent monomer segments. The poles are located on the $[0,0,1]$ lines of the lattice, that is, they are all parallel to the $z$ axis. In the plane normal to the $z$ axis they are assumed to be arranged regularly in $\S 1$, and in $\S 3$ they are assumed to be randomly arranged. In the regularly arranged case to be discussed in $\S 1$ the properties of the transition matrix connecting the probabilities of finding the $N$-th segment on a certain lattice point to those of finding the $(N+1)$-th segment on the adjacent lattice point are discussed in some detail. In $\S 2$ it is shown that the dependence of the average configurational properties of the polymers on the pole concentration, which is our main interest, can be calculated more easily in the randomly arranged case. The numerical results in the limit of large segment number are presented in $\S 3$ with some further discussion.

In this paper we use the word "adsorption" in an extended sense, including the repulsive (the positive adsorption energy) case. In addition we note that the word "free state" used 
here, which is often called the desorbed state, implies the non-adsorbed segment state.

\section{REGULARLY ARRANGED POLES}

We suppose that the regularly arranged poles are parallel to the $z$ axis and extend from $z=$ $-\infty$ to $z=+\infty$. Further, we assume that in the $x-y$ plane they are arranged in a twodimensional square lattice with the interpole distance $n l$, where $l$ is the length between adjacent segments of the polymer (we may easily extend the theory to other types of lattices). Let the adsorption energy between the pole and a segment be $-\varepsilon$; we define $\theta=\varepsilon / k_{\mathrm{B}} \mathbf{T}$, where $k_{\mathrm{B}}$ is the Boltzmann constant. We can easily apply the matrix method to this system. Suppose a simple cubic lattice whose lattice constant is $l$. Each segment of the polymer is located on a virtual lattice point, and each vector from one segment to the adjacent segment of the polymer takes the $x, y$, or $z$ direction with equal probability because of the assumed flexibility of the polymer chain. Since the adsorbing poles are the lines on the lattice parallel to the $z$ axis, the lattice probability of the segment should be multiplied by the factor $\alpha=\mathbf{e}^{\theta}$ when the segment lies on these adsorbing lines. Thus, if $P_{N}(i, j)$ is the unnormalized probability that the $N$-th segment of the polymer chain lies on the lattice point $(i, j)$, where $i, j$ designate the $x, y$ coordinates, the relation between $P_{N}(i, j)$ and $P_{N+1}(i, j)$ is written as follows (for the derivation see below):

$$
\mathbf{P}_{N+1}=\mathbf{T} \mathbf{P}_{N},
$$

where $\mathbf{P}_{N}$ and $\mathbf{P}_{N+1}$ are the following column vectors.

$$
\mathbf{P}_{N}=\left[\begin{array}{c}
P_{N}(0,0) \\
P_{N}(1,0) \\
\vdots \\
P_{N}(n-1,0) \\
P_{N}(0,1) \\
\vdots \\
P_{N}(n-1,1) \\
P_{N}(0,2) \\
\vdots \\
P_{N}(n-1, n-1)
\end{array}\right]
$$

$$
\mathbf{P}_{N+1}=\left[\begin{array}{c}
P_{N+1}(0,0) \\
P_{N+1}(1,0) \\
\vdots \\
P_{N+1}(n-1,0) \\
P_{N+1}(0,1) \\
\vdots \\
P_{N+1}(n-1,1) \\
P_{N+1}(0,2) \\
\vdots \\
P_{N+1}(n-1, n-1)
\end{array}\right],
$$

and

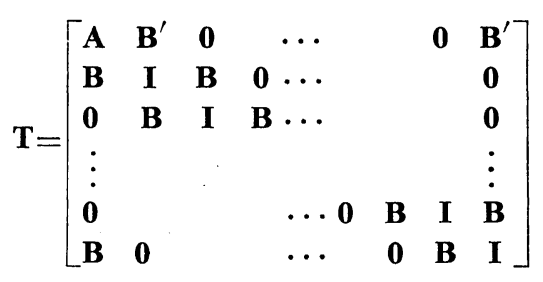

In the above the matrix $\mathbf{T}$ is of $n^{2} \times n^{2}$ type, while $\mathbf{A}, \mathbf{B}, \mathbf{B}^{\prime}, \mathbf{I}, \mathbf{0}$ are $n \times n$ matrices, since $\mathbf{T}$ relates the column vector $\mathbf{P}_{N+1}$ with $\mathbf{P}_{N}$, both of which have $n^{2}$ elements from $P(0,0)$ to $P(n-1$, $n-1$ ) (the intermediate elements are arranged as described in the r.h.s. of eq 2). In order to construct the matrix $\mathbf{T}$, it suffices to take into account the following relations. First, all probabilities $\mathbf{P}_{N+1}$ and $\mathbf{P}_{N}$ which have adjacent $(x, y)$ coordinates (for example $\mathbf{P}_{N+1}(1,3)$ and $\mathbf{P}_{N}(1,4)$ must be connected by the probability $\frac{1}{6}$. Secondly, $\mathbf{P}_{N+1}$ and $\mathbf{P}_{N}$ that have the same $(x, y)$ coordinates must be connected by the probability $\frac{1}{3}$, since the segment may be transported up or down along the $z$ axis. Thirdly, when the adsorbing line $(0,0)$ is occupied, $\alpha$ should be multiplied by the above probability, as mentioned before. Finally, we note that the $n^{2} \times n^{2}$ matrix $\mathbf{T}$ is enough to describe the relation between $P_{N+1}$ and $P_{N}$ even in the large segment number limit, because of the periodicity of the problem. For example, $P_{N+1}(n, n)$, the adsorbing site, can be identified with $P_{N+1}(0,0)$, $P_{N+1}(-1,0)$ can be identified with $P_{N+1}(n-1,0)$, etc., since what is essential to the present problem (in the large segment number limit) is the structure of the transition matrix $\mathbf{T}$. Then, from what we have stated, the $n \times n$ matrices $\mathbf{A}, \mathbf{I}, \mathbf{B}, \mathbf{B}^{\prime}, \mathbf{0}$ are written in the following forms. 


\section{J. Murakami}

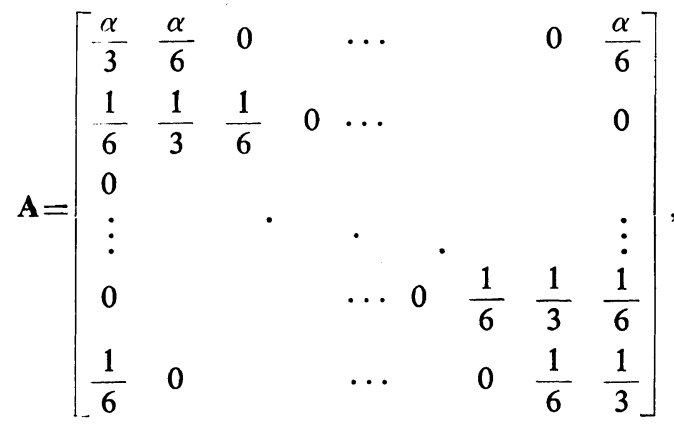

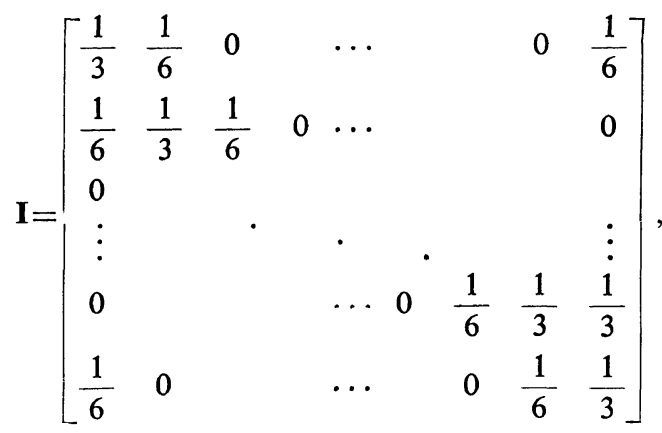

$$
\begin{aligned}
& \mathbf{B}=\left[\begin{array}{cccc}
\frac{1}{6} & 0 & \cdots & 0 \\
0 & \frac{1}{6} & & \\
\vdots & & \ddots & \vdots \\
0 & & & \frac{1}{6}
\end{array}\right]=\frac{1}{6} \mathbf{e} \\
& \mathbf{B}^{\prime}=\left[\begin{array}{cccc}
\frac{\alpha}{6} & 0 & \cdots & 0 \\
0 & \frac{1}{6} & & \\
\vdots & & \ddots & \vdots \\
0 & & & \frac{1}{6}
\end{array}\right]
\end{aligned}
$$

In the above, $\mathbf{e}$ is the $n \times n$ unit matrix and $\mathbf{B}^{\prime}$ has the same elements as $\mathbf{B}$ except that the latter $(1,1)$ element is replaced by $\alpha / 6$. I is a matrix in which the diagonal elements are $\frac{1}{3}$, their adjacent (end elements, e.g., $(i, 1)$ and $(i, n)$ are considered to be adjacent here) elements are $\frac{1}{6}$, and all other elements are $0 . \quad \mathbf{A}$ has the same elements as $\mathbf{I}$ except that the latter $(1,1)(1,2)(1, n)$ elements are replaced by $\alpha / 3, \alpha / 6, \alpha / 6$, respectively. $\mathbf{0}$ is a matrix whose elements are all 0 . In this way we have determined in the matrix $\mathbf{T}$.
When the segment number $N$ is very large, as is well known, the largest eigenvalue $\lambda_{m}$ of the matrix $\mathbf{T}$ rules the behavior of the partition function and the related equilibrium average quantities. The secular equation is written as follows ( $\mathbf{E}$ is the $n^{2} \times n^{2}$ unit matrix):

$$
\begin{aligned}
& \operatorname{det} \tilde{\mathbf{T}}=\operatorname{det}(\mathbf{T}-\lambda \mathbf{E})
\end{aligned}
$$

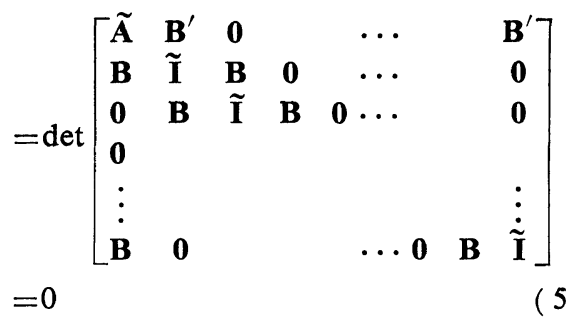

where $\tilde{\mathbf{A}}$ and $\tilde{\mathbf{I}}$ are $(\mathbf{A}-\lambda \mathbf{e})$ and $(\mathbf{I}-\lambda \mathbf{e})$, respectively.

The $n^{2} \times n^{2}$ determinant $\operatorname{det} \tilde{\mathbf{T}}$ is reduced to the $2 n \times 2 n$ determinant by the following procedure. For convenience, suppose that $\tilde{\mathbf{T}}$ is a $n \times n$ hypermatrix, each element also being a $n \times n$ matrix $\left(\tilde{\mathbf{A}}, \mathbf{B}, \mathbf{B}^{\prime}, \tilde{\mathbf{I}}\right.$ and $\left.\mathbf{0}\right)$. In the first column we sweep $\tilde{\mathbf{A}}$ in the first row and $\mathbf{B}$ in the $n$-th row.

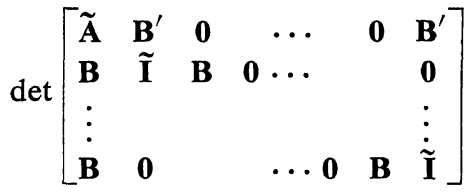

$$
\begin{aligned}
& =\operatorname{det}\left[\begin{array}{ccrrrr}
\mathbf{0} & \left(\mathbf{B}^{\prime}-6 \tilde{\mathbf{A}} \tilde{\mathbf{I}}\right) & -\tilde{\mathbf{A}} & \mathbf{0} & \ldots & \mathbf{B}^{\prime} \\
\mathbf{B} & \tilde{\mathbf{I}} & \mathbf{B} & \mathbf{0} & \ldots & \mathbf{0} \\
\vdots & & & & \vdots \\
\mathbf{0} & -\tilde{\mathbf{I}} & -\mathbf{B} & \mathbf{0} & \ldots & \dot{\tilde{\mathbf{I}}}
\end{array}\right]
\end{aligned}
$$

Since B is diagonal, the above is reduced to the following $(n-1) \times(n-1)$ determinant:

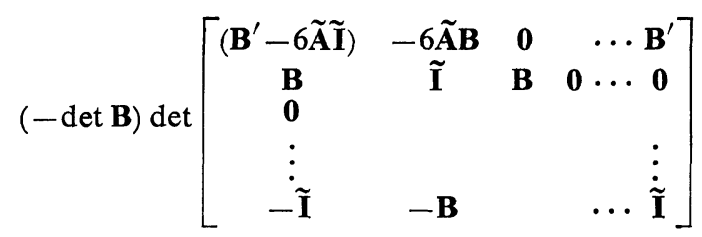

This has the same structure as the first $n \times n$ (hyper) matrix; therefore, it is possible to reduce this matrix to the $(n-2) \times(n-2)$ matrix by the method described above. Repeating this procedure we finally arrive at the $2 n \times 2 n$ determinant 


$$
(-\operatorname{det} \mathbf{B})^{n-2} \operatorname{det}\left[\begin{array}{c}
\varphi_{n-1}, \mathbf{B}^{\prime}-\boldsymbol{\varphi}_{n-2} \\
\mathbf{B}+\boldsymbol{\xi}_{n-1}, \tilde{\mathbf{I}}-\boldsymbol{\xi}_{n-2}
\end{array}\right]
$$

where $\varphi$ and $\xi$ satisfy the same recurrence formulas

$$
\begin{aligned}
& \varphi_{n}=-\varphi_{n-2}-6 \varphi_{n-1} \tilde{\mathbf{I}} \\
& \xi_{n}=-\xi_{n-2}-6 \xi_{n-1} \tilde{\mathbf{I}}
\end{aligned}
$$

with initial conditions

$$
\begin{array}{cc}
\varphi_{1}=\tilde{\mathbf{A}}, & \varphi_{0}=-\mathbf{B}^{\prime} \\
\boldsymbol{\xi}_{1}=\mathbf{B}, \quad \boldsymbol{\xi}_{0}=0
\end{array}
$$

Equations 8, 9, and 10 gives the secular equation,

$$
\operatorname{det}\left[\begin{array}{c}
\boldsymbol{\varphi}_{n-1}, \mathbf{B}^{\prime}-\boldsymbol{\varphi}_{n-2} \\
\mathbf{B}+\boldsymbol{\xi}_{n-1}, \tilde{\mathbf{I}}-\boldsymbol{\xi}_{n-2}
\end{array}\right]=0
$$

This is $n^{2}$-th order equation with respect to $\lambda$. It is awkward to obtain $\varphi_{n-1}, \varphi_{n-2}, \xi_{n-1}, \boldsymbol{\xi}_{n-2}$ by the recurrence formulas 9,10 , and to solve the $n^{2}$-th order equation to find the largest root $\lambda_{m}$, especially for large $n$. Thus, although we can solve the problem in principle and can construct the computational scheme, this becomes very complicated for large $n$ (dilute concentration of poles), e.g., even for $n=4$ we must solve a 16 -th order equation, in addition to the calculation of the matrix recurrence equations of several steps. This is inadequate for investigating the concentration dependence of various average properties.

Now let us turn to a case where a simple analytical treatment is possible.

\section{RANDOMLY ARRANGED POLES}

In this section we discuss the same problem as in the previous section except that in the present section the poles are distributed at randm in the $x-y$ plane. The mean concentration of the poles in this plane is $c$. We note that the present treatment can also be regarded as an approximation to that of the previous section, $1 /(n l)^{2}$ corresponding to $c$. This approximation, of couse, neglects the short range correlation (i.e., the lattice regularity in the present case) while preserving the long range property (cocentration $c$ ), which corresponds to the well-known mean field type approximation.

Suppose a virtual lattice (we consider a simple cubic lattice for simplicity) as before and consider a certatin lattice site called 0 . The ad- jacent four sites in the $x-y$ plane are called 1 , $2,3,4$, and the adjacent two sites in the $z$ direction may also called be 0 , because the energy state does not change in this direction. Let $P_{N}(i, A)$ and $P_{N}(i, F)$ be the respective probabilities of finding the $N$-th segment at the position $i$ of the adsorbed state $\left(P_{N}(i, A)\right)$ and of the free state $\left(P_{N}(i, F)\right)$. Then we obtatin of the following relation between $P_{N+1}$ and $P_{N} \cdot{ }^{8}$

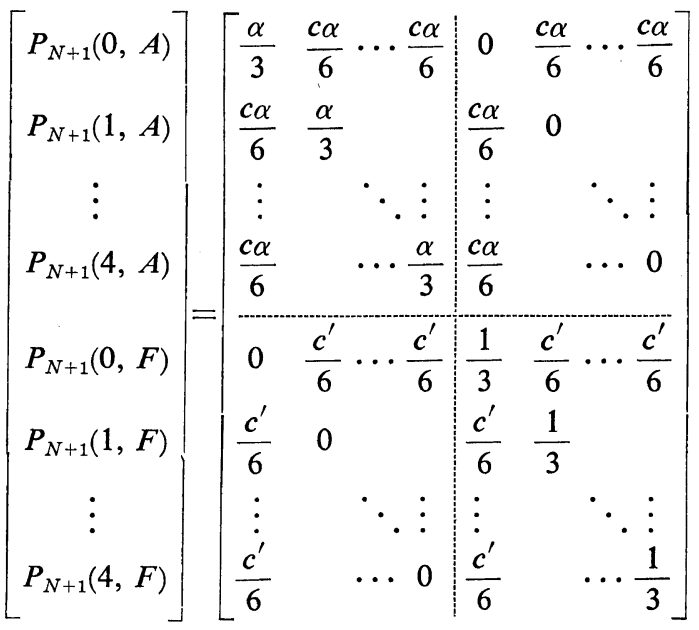

$$
\begin{aligned}
& \times\left[\begin{array}{c}
P_{N}(0, A) \\
P_{N}(1, A) \\
\vdots \\
P_{N}(4, A) \\
P_{N}(0, F) \\
P_{N}(1, F) \\
\vdots \\
P_{N}(4, F)
\end{array}\right]
\end{aligned}
$$

where $c^{\prime}=1-c, \alpha=e^{\theta}$, and we call the above $10 \times 10$ matrix $U$. Here we we have the assumption that whether the adjacent site is an adsorbing pole or not is always determined by the probability $c$ or $c^{\prime}$. The $10 \times 10$ matrix $\mathbf{U}$, corresponding to $\mathbf{T}$ in the previous section, determines the partition function $Z$, and for large $N$ the partition function is dominated by $\lambda_{m}$, the largest eigenvalue of $\mathbf{U}$ as $Z \approx \lambda_{m}{ }^{N}$. 


\section{J. Murakami}

Now, for the matrix $\mathbf{U}$, thanks to the assumption mentioned above, the secular equation is easily obtained by an elementary calculation,

$$
\begin{aligned}
\operatorname{det}|\mathbf{U}-\lambda \mathbf{E}|= & {\left[\lambda^{2}-\left(\frac{1}{6}+\frac{\alpha}{3}+\frac{c}{6}-\frac{c \alpha}{6}\right) \lambda+\frac{\alpha}{18}\right]^{4} } \\
& \times\left[\lambda^{2}-\left(1+\frac{\alpha}{3}+\frac{2 c \alpha}{3}-\frac{2 c}{3}\right) \lambda+\frac{\alpha}{3}\right] \\
= & 0
\end{aligned}
$$

where $\mathbf{E}$ is the $10 \times 10$ unit matrix. The largest root of this secular equation is always

$$
\begin{aligned}
\lambda_{m}= & \left\{1+\frac{\alpha}{3}+\frac{2 c \alpha}{3}-\frac{2 c}{3}\right. \\
& \left.+\left[\left(1+\frac{\alpha}{3}+\frac{2 c \alpha}{3}-\frac{2 c}{3}\right)^{2}-\frac{4 \alpha}{3}\right]^{1 / 2}\right\} / 2
\end{aligned}
$$

Then average equilibrium quantities are calculated from this as follows:

(1) Adsorbed Segment Ratio $\theta_{\mathrm{a}}{ }^{7,8}$

$$
\begin{aligned}
\theta_{\mathrm{a}}= & \frac{1}{N} \frac{\partial \ln Z}{\partial \ln \alpha} \\
= & \frac{\alpha}{2 \lambda_{m}}\left\{\frac{1}{3}+\frac{2 c}{3}\right. \\
& \left.+\frac{\left(1+\frac{\alpha}{3}+\frac{2 c \alpha}{3}-\frac{2 c}{3}\right)\left(\frac{1+2 c}{3}\right)-\frac{2}{3}}{\left[\left(1+\frac{\alpha}{3}+\frac{2 c \alpha}{3}-\frac{2 c}{3}\right)^{2}-\frac{4 \alpha}{3}\right]^{1 / 2}}\right\}
\end{aligned}
$$

(2) Sequence Number per Segment $\nu$

In order to count the sequence number we introduce the dummy counting variable $g$ into lower left quarter of the matrix $U$. Then a $g$ factor appears when the segment changes from the adsorbed state to the free state, therefore, this factor counts the number of sequences. If we solve the secular equation for this modified matrix $\mathbf{U}_{g 1}$,

$$
\mathbf{U}_{g 1}=\left[\begin{array}{ccc:ccc}
\frac{\alpha}{3} & \cdots & \frac{c \alpha}{6} & 0 & \cdots & \frac{c \alpha}{6} \\
\vdots & \ddots & \vdots & \vdots & \ddots & \vdots \\
\frac{c \alpha}{6} & \cdots & \frac{\alpha}{3} & \frac{c \alpha}{6} & \cdots & 0 \\
\hdashline 0 & \cdots & \frac{g c^{\prime}}{6} & \frac{1}{3} & \cdots & \frac{c^{\prime}}{6} \\
\vdots & \ddots & \vdots & \vdots & \ddots & \vdots \\
\frac{s c^{\prime}}{6} & \cdots & 0 & \frac{c^{\prime}}{6} & \cdots & \frac{1}{3}
\end{array}\right]
$$

$$
\begin{aligned}
\operatorname{det}\left(\mathbf{U}_{g 1}-\lambda \mathbf{E}\right) & =\left\{\left[\lambda^{2}-\left(\frac{1}{6}+\frac{\alpha}{3}+\frac{c}{6}-\frac{c \alpha}{6}\right) \lambda+\frac{\alpha}{18}\right]\right. \\
& \left.-\frac{(g-1) c c^{\prime} \alpha}{36}\right\}^{4}\left\{\left[\lambda^{2}-\left(1+\frac{\alpha}{3}+\frac{2 c \alpha}{3}-\frac{2 c}{3}\right) \lambda\right.\right. \\
& \left.\left.+\frac{\alpha}{3}\right]-\frac{(g-1) c c^{\prime} \alpha}{36}\right\}=0,
\end{aligned}
$$

we may obtain $\nu$ by differentiating $\ln Z$ with respect to $\ln g$ and dividing the result by $N,{ }^{7}$

$$
\begin{aligned}
\nu & =\frac{1}{N} \frac{\partial \ln Z}{\partial \ln g}=-\left.\left.\frac{\partial \ln \lambda_{m}}{\partial \ln \alpha}\right|_{g=1} \frac{\partial \ln \alpha}{\partial \ln g}\right|_{\lambda_{m}} \\
& =\frac{c c^{\prime} \theta_{\mathrm{a}}}{12\left[(1+2 c) \lambda_{m}-\frac{1}{3}\right]}
\end{aligned}
$$

(3) Average Length of the Adsorbed $\left(j_{\mathrm{a}}\right)$ and Free $\left(j_{\mathrm{f}}\right)$ Sequences

The average length of the absorbed sequence $j_{\mathrm{a}}$ and that of the free sequence $j_{\mathrm{f}}$ are obtained from $\theta_{\mathrm{a}}$ and $\nu$ as follows: ${ }^{7}$

$$
\begin{gathered}
j_{\mathrm{a}}=\theta_{\mathrm{a}} / \nu \\
j_{\mathrm{f}}=\left(1-\theta_{\mathrm{a}}\right) / \nu
\end{gathered}
$$

$j_{\mathrm{a}}$ and $j_{\mathrm{f}}$ here are the number-average lengths. We may define $j_{\mathrm{a}}$ and $j_{\mathrm{f}}$ in other ways. But the characteristic behavior of the sequence length will be well represented by the above quantities.

Now we will numerically evaluate $\theta_{\mathrm{a}}, \nu, j_{\mathrm{a}}$, and $j_{\mathrm{f}}$ in the following section. Other equilibrium quantities such as the distribution of the sequence lengths and the free energy of the system can be derived by a similar procedure.

\section{NUMERICAL RESULTS AND DISCUSSION}

In Figure 1 the fraction of the adsorbed segment $\theta_{\mathrm{a}}$ is plotted against the adsorption energy $\theta$ for the pole concentrations $c=0.2,0.5$, and 0.75. Of course, the curves pass through $\theta_{\mathrm{a}}=$ $0.2,0.5$, and 0.75 at $\theta=0$, respectively. In Figure 2 the sequence number per segment is plotted against $\theta$. Each curve shows a maximum, because at larger values of $|\theta|$ long sequences appear (as is shown in Figures 3 and 4) and in consequence the sequence number decreases. The value of $\theta$ at which the sequence number $\nu$ become maximum gradually increases as the pole concentration becomes smaller (the maximum occurs at $\theta=-0.22,0.26,0.89$ when 


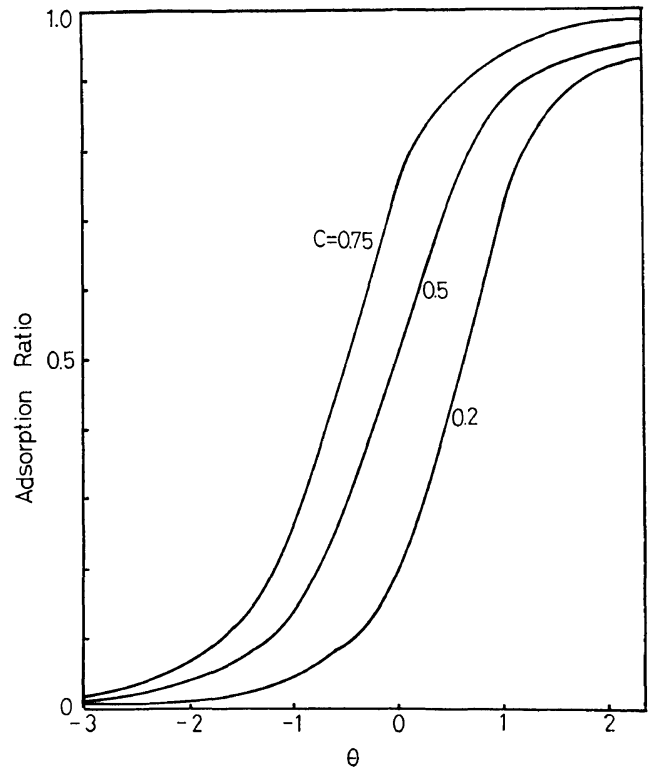

Figure 1. The adsorbed segment ratio $\theta_{\mathrm{a}}$ is plotted $v s$. adsorption energy $\theta$ for concentrations $c=0.2$, $0.5,0.75$.

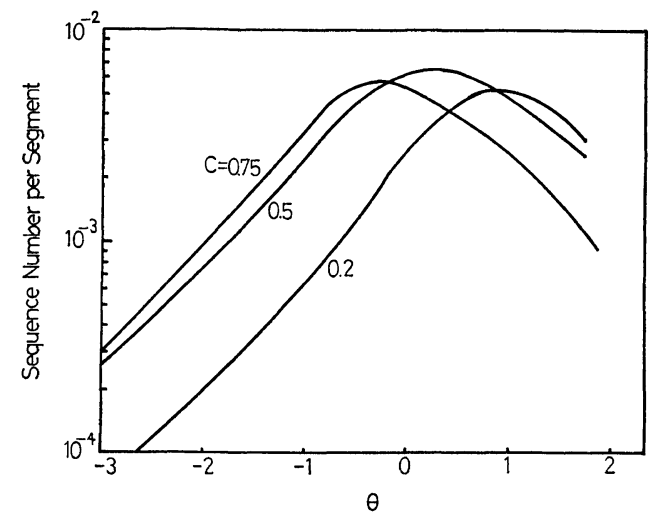

Figure 2. The sequence number per segment $\nu$ is plotted $v s$. adsorption energy $\theta$ for concentrations $c=0.2,0.5,0.75$.

$c=0.75,0.5,0.2$, respectively), and the maximum $\nu$ value is largest for $c=0.5$ (maximum $\nu$ is $5.6 \times 10^{-3}, 6.5 \times 10^{-3}, 5.2 \times 10^{-3}$, when $c=0.75$, $0.5,0.2$, respectively).

In consequence, at the repulsive $\theta$ the sequence length in the free state becomes very large as $c$ decreases, while at the attractive $\theta$ the sequence length in the adsorbed state grows as $c$ increases. These behaviors are shown in Figures 3 and 4.

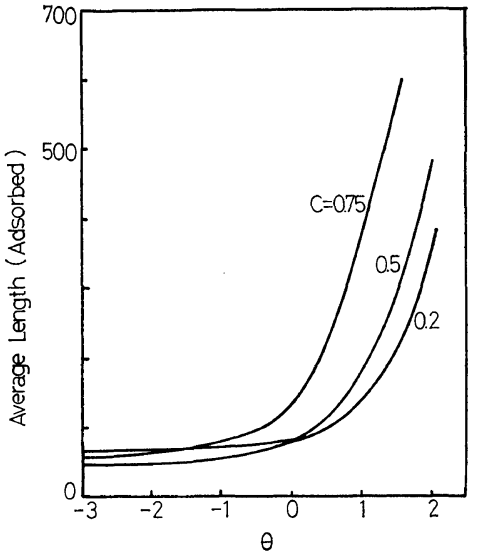

Figure 3. Average length of the adsorbed sequece $j_{\mathrm{a}}$ is plotted $v s$. adsorption energy $\theta$ for concentrations $c=0.2,0.5,0.75$.

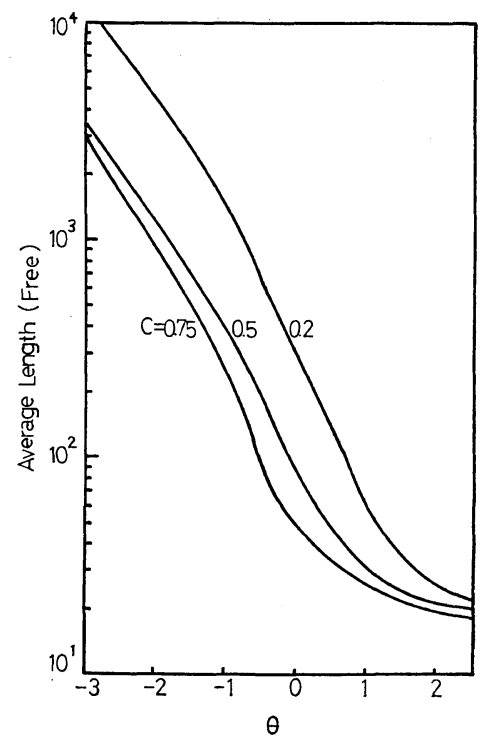

Figure 4. Average length of the free sequence $j_{\mathrm{f}}$ is plotted $v s$. adsorption energy $\theta$ for concentrations $c=0.2,0.5,0.75$.

On the other hand, the length of the free sequence at the attractive $\theta$ and the length of the adsorbed sequence at the repulsive $\theta$ are rather insensitive to the pole concentration.

It should be stressed that although we can understand from an intuitive physical basis the qualitative behavior of the above-mentioned average quantities characterizing the polymer configuration, quantitative results are obtainable 


\section{J. MuraKami}

only when the detailed calculation is actually performed.

Now, let us discuss the basic assumptions and the qualitative and/or quantitative appropriateness of the above calculations. The first assumption, although not explicitly stated in the previous section, is that we have replaced the adsorption which should be an interfacial phenomenon by a simple point-like interaction with the energy change $-\varepsilon$. This assumption, however, will be permissible in our present situation, since here the (pole) concentration dependences of various average configurational properties of the polymer are of interest.

The second assumption is the mean filed approximation stated in the previous section. This approximation discards the detailed information about the position of the adsorbing poles, that is, whether a certain lattice site in the $x-y$ plane is an adsorbing pole or not changes step by step with the segment of the polymer, only conserving the mean probabilities $c$ (adsorbing case) and $c^{\prime}=1-c$ (free case) and the fundamental requirements of the nearest-neighbor transitions (as described by the matrix $\mathbf{T}$ in the previous section). To investigate the adequateness of this approximation, it is of interest to calculate a regularly arranged case where $n=2 \quad\left(c=\frac{1}{4}\right)$ and to compare the result with that of the randomly arranged case where $c=\frac{1}{4}$.

Though the matrix $\mathbf{T}$ in eq 1 is complicated in general as stated in $\S 1$, it takes a simple form in this special case:

$$
\mathbf{T}=\left[\begin{array}{cccc}
\frac{\alpha}{3} & \frac{\alpha}{6} & \frac{\alpha}{6} & 0 \\
\frac{1}{6} & \frac{1}{3} & 0 & \frac{1}{6} \\
\frac{1}{6} & 0 & \frac{1}{3} & \frac{1}{6} \\
0 & \frac{1}{6} & \frac{1}{6} & \frac{1}{3}
\end{array}\right]
$$

Then the secular equation is easily obtained as

$$
\lambda\left(\lambda-\frac{1}{3}\right)\left(\lambda^{2}-\frac{\alpha+2}{3} \lambda+\frac{3 \alpha+1}{18}\right)=0
$$

and its largest root is

$$
\lambda_{m}=\frac{\alpha+2+\left(\alpha^{2}-2 \alpha+2\right)^{1 / 2}}{6}
$$

From this average quantities are obtained as in $\S 2$,

$$
\begin{gathered}
\theta_{\mathrm{a}}=\frac{\alpha}{6 \lambda_{m}}\left[1+\frac{\alpha-1}{\left(\alpha^{2}-2 \alpha+2\right)^{1 / 2}}\right] \\
\nu=\theta_{\mathrm{a}} \frac{\lambda_{m}-\alpha / 3}{\lambda_{m}}
\end{gathered}
$$

Since $j_{\mathrm{a}}$ and $j_{\mathrm{f}}$ are determined from these two quantities, we discuss only these two values. From the comparison of the numerical results of the above formulas with those of the randomly arranged case $\left(c=\frac{1}{4}\right)$, we are led to the following observations:

(1) The adsorption ratio $\theta_{\mathrm{a}}$ agrees quite well in both cases over the entire energy range. The relative difference is of the order $10^{-2}$.

(2) Contrary to the adsorption ratio $\theta_{\mathrm{a}}$, the sequence number $\nu$ in the regularly arranged case is usually $10-30$ times larger than that in the randomly arranged case, and therefore, $j_{\mathrm{a}}$ and $j_{\mathrm{f}}$ differ in the same ratio but in the inverse order. However, the overall qualitative behaviors are the same (the maximum of $\nu$, that is, $1.6 \times 10^{-1}$ appears when $\theta=0.55$ in the regularly arranged case, whereas the maximum of $\nu$ is $5.7 \times 10^{-3}$ when $\theta=0.78$ in the randomly arranged case).

Since the regularly and randomly arranged cases are different, the results need not be in complete agreement. But the disagreement found in (2) seems to be a significant one, and it arises from the mean field approximation mentioned above. If we take a matrix of larger size than that considered in $\S 2$ (e.g., taking into account the second neighbors, the third neighbors, ...), an improvement may be accomplished, although the calculations will be more and more complicated. These are interesting problems, but here we do not pursue them further and leave them for future investigations.

In conclusion, from the above comparison and considerations, $\theta_{\mathrm{a}}$ calculatedi $\mathrm{n} \S 2$ will be fairly accurate in spite of the above-mentioned assumptions, but $\nu, j_{\mathrm{a}}$ and $j_{\mathrm{f}}$ calculated in $\S 2$ will not be sufficiently accurate, since these quantities are closely dependent on the information discarded by the mean field approximation. However it should be stressed that the present theory provides the simplest quantitatively tractable approximation, and that the results show physi- 
cally cosistent qualitative behaviors over all concentrations of the adsorbing poles.

Finally, we discuss the possible generalizations of the present theory. First, in order to approach a more realistic situation, the stiffness of the polymer and the excluded volume effect should be taken into account. These effects have been subjected to some investigations in the case of the polymer adsorbed by one plane wall, ${ }^{2,7,8}$ but it seems rather difficult in the parallel plane wall systems as well as in the present case of a distributed pole system.

Another generalization is the case of a system in which many kinds of poles exist. This problem may be easily treated by slightly extending the present approach. For example, when two kinds of poles exist, the concentrations being $c_{1}$ and $c_{2}$, the equation corresponding to eq 12 is written as

$$
\left[\begin{array}{c}
P_{N+1}\left(0, A_{1}\right) \\
\vdots \\
P_{N+1}\left(4, A_{1}\right) \\
P_{N+1}\left(0, A_{2}\right) \\
\vdots \\
P_{N+1}\left(4, A_{2}\right) \\
P_{N+1}(0, F) \\
\vdots \\
P_{N+1}(4, F)
\end{array}\right]=\left[\begin{array}{ccc:ccc:ccc}
\frac{\alpha_{1}}{3} & \cdots & \frac{c_{1} \alpha_{1}}{6} & 0 & \cdots & \frac{c_{1} \alpha_{1}}{6} & 0 & \cdots & \frac{c_{1} \alpha_{1}}{6} \\
\vdots & \ddots & \vdots & \vdots & \ddots & \vdots & \vdots & \ddots & \vdots \\
\frac{c_{1} \alpha_{1}}{6} & \cdots & \frac{\alpha_{1}}{3} & \frac{c_{1} \alpha_{1}}{6} & \cdots & 0 & \frac{c_{1} \alpha_{1}}{6} & \cdots & 0 \\
\hdashline 0 & \cdots & \frac{c_{2} \alpha_{2}}{6} & \frac{\alpha_{2}}{3} & \cdots & \frac{c_{2} \alpha_{2}}{6} & 0 & \cdots & \frac{c_{2} \alpha_{2}}{6} \\
\vdots & \ddots & \vdots & \vdots & \ddots & \vdots & \vdots & \ddots & \vdots \\
\frac{c_{2} \alpha_{2}}{6} & \cdots & 0 & \frac{c_{2} \alpha_{2}}{6} & \cdots & \frac{\alpha_{2}}{3} & \frac{c_{2} \alpha_{2}}{6} & \cdots & 0 \\
\hdashline 0 & \cdots & \frac{c_{3}}{6} & 0 & \cdots & \frac{c_{3}}{6} & \frac{1}{3} & \cdots & \frac{c_{3}}{6} \\
\vdots & \ddots & \vdots & \vdots & \ddots & \vdots & \vdots & \ddots & \vdots \\
\frac{c_{3}}{6} & \cdots & 0 & \frac{c_{3}}{6} & \cdots & 0 & \frac{c_{3}}{6} & \cdots & \frac{1}{3}
\end{array}\right]\left[\begin{array}{c}
P_{N}\left(0, A_{1}\right) \\
\vdots \\
P_{N}(4, F)
\end{array}\right]
$$

where $A_{1}, A_{2}$ and $F$ in the brackets designate the adsorbed states with energy $-\varepsilon_{1}\left(A_{1}\right),-\varepsilon_{2}\left(A_{2}\right)$, and free state $(F)$ respectively, and $\alpha_{1}=\exp \left(\varepsilon_{1} / k_{\mathrm{B}} \mathbf{T}\right)$, $\alpha_{2}=\exp \left(\varepsilon_{2} / k_{\mathrm{B}} \mathbf{T}\right), c_{3}=1-c_{1}-c_{2}$. Average quantities may be easily computed as a function of the parameters $\alpha_{1}, \alpha_{2}, c_{1}, c_{2}$, as in the previous section.

Acknowledgment. The author would like to thank Professor K. Okano for reading the manuscript and for giving valuable advice.

\section{REFERENCES}

1. W. C. Forsman and R. E. Hughes, J. Chem. Phys., 38, 2118, 2123, 2130 (1963).

2. A. Silberberg, J. Phys. Chem., 66, 1872, 1884 (1962); J. Chem. Phys., 46, 1105 (1967); ibid., 48,
2835 (1968).

3. E. A. DiMarzio, J. Chem. Phys., 42, 2101 (1965).

4. C. A. J. Hoeve, E. A. DiMarzio, and P. Peyser, ibid., 42, 2558 (1965).

5. E. A. DiMarzio and F. L. McCrackin, ibid., 43, 539 (1965).

6. C. A. J. Hoeve, ibid., 43, 3007 (1965).

7. R. J. Roe, Proc. Natl. Acad. Sci., 53, 50 (1965); J. Chem. Phys., 43, 1591 (1965); ibid., 44, 4264 (1966).

8. R. J. Rubin, J. Chem. Phys., 43, 2392 (1965); J. Res. Natl. Bur. Stnd., 69B, 301 (1965).

9. K. Motomura and R. Matuura, J. Chem. Phys., 50, 1281 (1969).

10. M. Lax, ibid., 61, 4133 (1974).

11. E. A. DiMarzio and R. J. Rubin, ibid., 55, 4318 (1971).

12. R. J. Rubin, ibid., 44, 2130 (1966). 